\title{
Correction to: De novo profiling of RNA viruses in Anopheles malaria vector mosquitoes from forest ecological zones in Senegal and Cambodia
}

Eugeni Belda ${ }^{1,2,3}$, Ferdinand Nanfack-Minkeu ${ }^{1,2,4}$, Karin Eiglmeier ${ }^{1,2}$, Guillaume Carissimo ${ }^{5}$, Inge Holm ${ }^{1,2}$, Mawlouth Diallo ${ }^{6}$, Diawo Diallo ${ }^{6}$, Amélie Vantaux ${ }^{7}$, Saorin Kim, Igor V. Sharakhov ${ }^{8}$ and Kenneth D. Vernick ${ }^{1,2^{*}}$

\section{Correction to: BMC Genomics \\ https://doi.org/10.1186/s12864-019-6034-1}

Following the publication of this article [1], the authors reported that the original shading in columns 3 and 4 of Table 3, which indicated the presence or absence of viruses in each library, had been removed during typesetting.

A corrected Table 3 indicating the presence or absence of viruses is included in this Correction article. The publisher would like to apologize to the authors and any readers for the inconvenience.

\footnotetext{
Author details

'Unit of Insect Vector Genetics and Genomics, Department of Parasites and Insect Vectors, Institut Pasteur, Paris, France. ${ }^{2}$ CNRS Unit of Evolutionary Genomics, Modeling, and Health (UMR2000), Institut Pasteur, Paris, France. ${ }^{3}$ Integromics Unit, Institute of Cardiometabolism and Nutrition, Assistance Publique Hôpitaux de Paris, Pitié-Salpêtrière Hospital, Paris, France. ${ }^{4}$ Sorbonne Université, Graduate School of Life Sciences ED515, UPMC - Université Pierre et Marie Curie - Paris 6, 4 Place Jussieu, 75252 Paris, France. ${ }^{5}$ Laboratory of Microbial Immunity, Singapore Immunology Network, Agency for Science, Technology and Research (A(*)STAR), Singapore, Singapore. Institut Pasteur de Dakar, Dakar, Senegal. ${ }^{7}$ Institut Pasteur of Cambodia, Phnom Penh, Cambodia. ${ }^{8}$ Department of Entomology, Virginia Polytechnic Institute and State University, Blacksburg, VA, USA.
}

Published online: 05 September 2019

\section{Reference}

1. Belda, et al. De novo profiling of RNA viruses in Anopheles malaria vector mosquitoes from forest ecological zones in Senegal and Cambodia. BMC Genomics. 2019;20:664 https://doi.org/10.1186/s12864-019-6034-1.

\footnotetext{
* Correspondence: kvernick@pasteur.fr

'Unit of Insect Vector Genetics and Genomics, Department of Parasites and Insect Vectors, Institut Pasteur, Paris, France

${ }^{2}$ CNRS Unit of Evolutionary Genomics, Modeling, and Health (UMR2000),

Institut Pasteur, Paris, France

Full list of author information is available at the end of the article
}

(c) The Author(s). 2019 Open Access This article is distributed under the terms of the Creative Commons Attribution 4.0 International License (http://creativecommons.org/licenses/by/4.0/), which permits unrestricted use, distribution, and reproduction in any medium, provided you give appropriate credit to the original author(s) and the source, provide a link to the Creative Commons license, and indicate if changes were made. The Creative Commons Public Domain Dedication waiver (http://creativecommons.org/publicdomain/zero/1.0/) applies to the data made available in this article, unless otherwise stated. 
Table 3 Similarity of Senegal and Cambodia virus assemblies by BLASTX to 24 reference viruses in GenBank. 10 targets are shared, 9 are Senegal-specific, and 5 are Cambodia-specific

\begin{tabular}{|c|c|c|c|}
\hline Reference virus & Viral taxonomy & Senegal libraries & Cambodia libraries \\
\hline $\begin{array}{l}\text { Culex tritaeniorhynchus rhabdovirus RNA, } \\
\text { complete genome }\end{array}$ & $\begin{array}{l}\text { Viruses; ssRNA viruses; ssRNA negative-strand viruses; } \\
\text { Mononegavirales; Rhabdoviridae; unclassified Rhabdoviridae. }\end{array}$ & Present & Present \\
\hline Phasi Charoen-like virus & $\begin{array}{l}\text { Viruses; ssRNA viruses; ssRNA negative-strand viruses; } \\
\text { Bunyavirales; unclassified Bunyavirales. }\end{array}$ & Present & Present \\
\hline Uncultured virus isolate acc_1.3 & Viruses; environmental samples. & Present & Present \\
\hline Wellfleet Bay virus isolate 10-280-G segment 4 & $\begin{array}{l}\text { Viruses; ssRNA viruses; ssRNA negative-strand viruses; } \\
\text { Orthomyxoviridae; Quaranjavirus; unclassified Quaranjavirus. }\end{array}$ & Present & Present \\
\hline Wuhan Mosquito Virus 1 strain WT3-15 & $\begin{array}{l}\text { Viruses; ssRNA viruses; ssRNA negative-strand viruses; } \\
\text { unclassified ssRNA negative-strand viruses. }\end{array}$ & Present & Present \\
\hline Wuhan Mosquito Virus 9 strain JX1-13 & $\begin{array}{l}\text { Viruses; ssRNA viruses; ssRNA negative-strand viruses; } \\
\text { unclassified ssRNA negative-strand viruses. }\end{array}$ & Present & Present \\
\hline Xincheng Mosquito Virus strain XC1-6 & $\begin{array}{l}\text { Viruses; ssRNA viruses; ssRNA negative-strand viruses; } \\
\text { unclassified ssRNA negative-strand viruses. }\end{array}$ & Present & Present \\
\hline Xinzhou Mosquito Virus strain XC3-5 & $\begin{array}{l}\text { Viruses; ssRNA viruses; ssRNA negative-strand viruses; } \\
\text { unclassified ssRNA negative-strand viruses. }\end{array}$ & Present & Present \\
\hline Beaumont virus strain 6 & $\begin{array}{l}\text { Viruses; ssRNA viruses; ssRNA negative-strand viruses; } \\
\text { Mononegavirales; Rhabdoviridae; unclassified Rhabdoviridae. }\end{array}$ & Present & Present \\
\hline Jurona virus & $\begin{array}{l}\text { Viruses; ssRNA viruses; ssRNA negative-strand viruses; } \\
\text { Mononegavirales; Rhabdoviridae; Vesiculovirus. }\end{array}$ & Present & Present \\
\hline Omono River virus & Viruses; dsRNA viruses & Present & Absent \\
\hline American dog tick phlebovirus isolate $\mathrm{Fl} 3$ & $\begin{array}{l}\text { Viruses; ssRNA viruses; ssRNA negative-strand viruses; } \\
\text { Bunyavirales; Phlebovirus; unclassified Phlebovirus. }\end{array}$ & Present & Absent \\
\hline Daeseongdong virus 1 strain A12.2708/ROK/2012 & Viruses; unclassified viruses. & Present & Absent \\
\hline $\begin{array}{l}\text { DsRNA virus environmental sample clone } \\
\text { mill.culi_contig84 }\end{array}$ & Viruses; dsRNA viruses; environmental samples. & Present & Absent \\
\hline Homalodisca vitripennis reovirus segment S3 & $\begin{array}{l}\text { Viruses; dsRNA viruses; Reoviridae; Sedoreovirinae; } \\
\text { Phytoreovirus; unclassified Phytoreovirus }\end{array}$ & Present & Absent \\
\hline $\begin{array}{l}\text { Ixodes scapularis associated virus } 2 \text { isolate A1, } \\
\text { partial genome }\end{array}$ & Viruses; unclassified viruses. & Present & Absent \\
\hline Sunn-hemp mosaic virus & $\begin{array}{l}\text { Viruses; ssRNA viruses; ssRNA positive-strand viruses, } \\
\text { no DNA stage; Virgaviridae; Tobamovirus. }\end{array}$ & Present & Absent \\
\hline Uncultured virus isolate acc_7.4 & Viruses; environmental samples. & Present & Absent \\
\hline Wuhan Spider Virus strain SYZZ-2 & $\begin{array}{l}\text { Viruses; ssRNA viruses; ssRNA negative-strand viruses; } \\
\text { unclassified ssRNA negative-strand viruses. }\end{array}$ & Present & Absent \\
\hline Nienokoue virus isolate B51/Cl/2004 & $\begin{array}{l}\text { Viruses; ssRNA viruses; ssRNA positive-strand viruses, } \\
\text { no DNA stage; Flaviviridae }\end{array}$ & Absent & Present \\
\hline Oat golden stripe virus RNA1 & $\begin{array}{l}\text { Viruses; ssRNA viruses; ssRNA positive-strand viruses, } \\
\text { no DNA stage; Virgaviridae; Furovirus }\end{array}$ & Absent & Present \\
\hline Puerto Almendras virus isolate LO-39 & $\begin{array}{l}\text { Viruses; ssRNA viruses; ssRNA negative-strand viruses; } \\
\text { Mononegavirales; Rhabdoviridae; unclassified Rhabdoviridae. }\end{array}$ & Absent & Present \\
\hline $\begin{array}{l}\text { Tobacco streak virus isolate pumpkin } \\
\text { segment RNA1 }\end{array}$ & $\begin{array}{l}\text { Viruses; ssRNA viruses; ssRNA positive-strand viruses, } \\
\text { no DNA stage; Bromoviridae; llarvirus }\end{array}$ & Absent & Present \\
\hline Bivens Arm virus isolate UF 10 & $\begin{array}{l}\text { Viruses; ssRNA viruses; ssRNA negative-strand viruses; } \\
\text { Mononegavirales; Rhabdoviridae; Tibrovirus }\end{array}$ & Absent & Present \\
\hline
\end{tabular}

